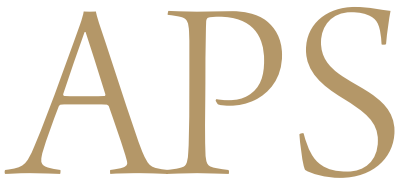

Archives of Plastic Surgery

\title{
A Multicenter Noncomparative Clinical Study on Midface Rejuvenation Using a Nonabsorbable Polypropylene Mesh: Evaluation of Efficacy and Safety
}

\author{
Chang Sik Pak ${ }^{1}$, Lan Sook Chang ${ }^{1}$, Hobin Lee ${ }^{1}$, Jae Hoon Jeong ${ }^{1}$, Jinwook Jeong ${ }^{1}$, \\ Eul-Sik Yoon ${ }^{2 *}$, Chan Yeong Heo ${ }^{1 *}$ \\ ${ }^{I}$ Department of Plastic and Reconstructive Surgery, Seoul National University Bundang Hospital, Seoul National University College of \\ Medicine, Seongnam; ${ }^{2}$ Department of Plastic and Reconstructive Surgery, Korea University Anam Hospital, Korea University College of \\ Medicine, Seoul, Korea
}

Background Facial rejuvenation can be achieved using a variety of techniques. Since minimally invasive procedures for face lifting have become popular because of their convenience and short operating time, numerous minimally invasive surgical procedures have been developed. In this study, a nonabsorbable polypropylene mesh is introduced as a new face lifting instrument, with the nasolabial fold as the main target area. In this paper, we report the efficacy and safety of a polypropylene mesh in midface rejuvenation.

Methods Thirty-three subjects with moderate-to-severe nasolabial folds were enrolled from two medical institutions for a noncomparative single-sample study. A mesh was inserted above the superficial muscular aponeurotic system layer, reaching the nasolabial folds through a temporal scalp incision. After 3 weeks, the temporal end of the mesh was pulled to provide a lifting effect. Then, the mesh was fixed to the deep temporal fascia using nonabsorbable sutures. To evaluate efficacy, we compared the scores on the Wrinkle Severity Rating Scale and a visual analog scale for patient satisfaction between the baseline and 7 weeks postoperatively. In addition, we evaluated safety based on the incidence of adverse events.

Results The treatment was deemed effective at improving wrinkles in 23 of 28 cases, and patient satisfaction improved significantly during the study period. There were seven cases of skin or subcutaneous tissue complications, including edema and erythema, but there were no suspected serious adverse events.

Conclusions Face lifting using a nonabsorbable mesh can improve nasolabial folds without serious adverse effects. Thus, this technique is safe and effective for midface rejuvenation.

Keywords Polypropylene / Rhytidoplasty / Facelift
Co-Correspondence: Chan Yeong Heo Department of Plastic and Reconstructive Surgery, Seoul National University Bundang Hospital, Seoul National University College of Medicine, 82 Gumi-ro 173beon-gil, Bundang-gu, Seongnam 13620, Korea Tel: +82-31-787-7222

Fax: +82-31-787-4055

E-mail: lionheo@snu.ac.kr

\section{Eul-Sik Yoon}

Department of Plastic and Reconstructive Surgery, Korea University Anam Hospital, Korea University College of Medicine, 73 Inchon-ro, Seoungbuk-gu, Seoul 02841, Korea

Tel: +82-2-922-7437

Fax: +82-2-920-6744

E-mail: yesanam2@kumc.or.kr

${ }^{*}$ Chan Yeong Heo and Eul-Sik Yoon have contributed equally to this work.

No potential conflict of interest relevant to this article was reported. 


\section{INTRODUCTION}

Facial rejuvenation has been popular for decades, from simple noninvasive procedures to more invasive surgical treatments. Among numerous current noninvasive procedures, fillers and botulinum toxin are used worldwide. However, these simple procedures require repeated treatments to maintain their effects. Although conventional face lifting surgery can guarantee more lasting effects, the burden of such an invasive operation is large. Consequently, many face lifting alternatives have been developed. In Korea, various face lifting alternatives, such as thread lift, gold thread implantation, Happy Lift, and feather lift, have become popular [1-3]. For example, by using nonabsorbable polypropylene barbed thread, a semipermanent face lifting effect can be achieved by pulling the thread and fixing it in the temporal area [3-5]. Thread lifting, through the body's reaction to the inserted foreign substances, uses the tension of the thread to remove wrinkles and promote collagen regeneration; in this technique, absorbable or nonabsorbable thread can be used. Silhouette lifting - another popular type of minimally invasive face lifting surgery - consists of a cone with the components of glycolic acid and lactic acid that supports the skin and soft tissue, as well as nonabsorbable polypropylene thread [6]. Although thread lifting has many advantages, including its simple procedure and short recovery time, it has limited face lifting effects because of the small contact area with the skin tissue. Mutaf [7] reported the use of a polypropylene mesh as an alternative to brow lifting in 37 patients with brow ptosis. During the follow-up period of 4 years and 6 months, there were no adverse effects of foreign body insertion and no recurrence of brow ptosis. We have designed mesh lifting to achieve a larger contact area with the skin and induce greater face lifting effects. Further, we have designed this clinical study to report the lifting and rejuvenation effects of a nonabsorbable mesh, which had not yet been clarified.

\section{METHODS}

\section{Statement of ethics}

This clinical trial was approved by the institutional review boards of each investigational institution. Patients were informed about the research protocol both verbally and in writing before they were asked to participate in this study and before they signed an informed consent form.

\section{Study subjects}

This is a multicenter noncomparative single-sample clinical study. The study was conducted at two medical institutions between January 2013 and December 2013. We initially recruited a total

\section{Table 1. Inclusion and exclusion criteria}

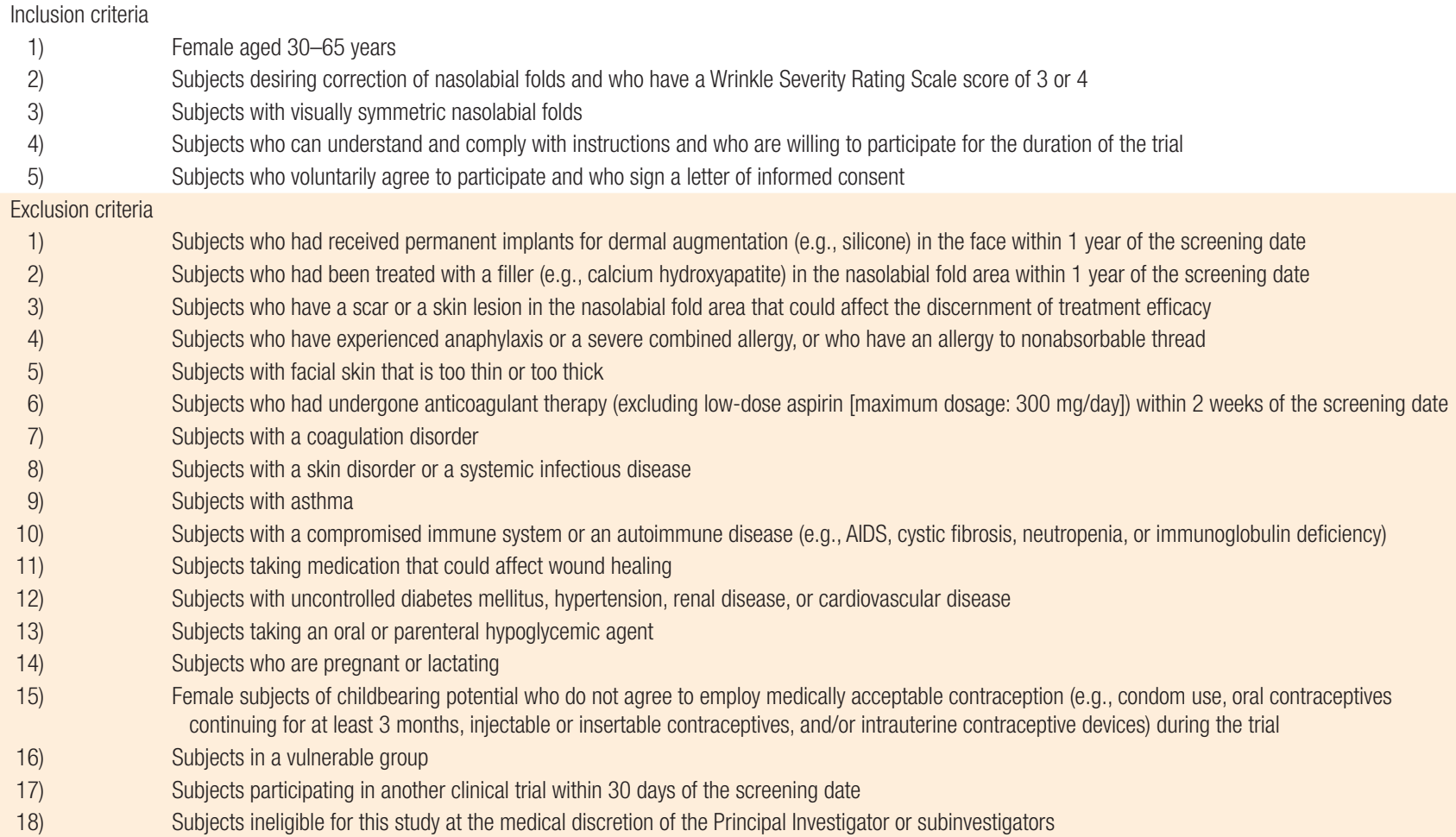


of 33 subjects, but excluded 4 through baseline screening. The inclusion and exclusion criteria are shown in Table 1. One patient also withdrew written informed consent, resulting in a final enrollment of 28 subjects in the current study. All patients provided written informed consent, and all completed the current study. The experimental side nasolabial fold was selected by using a random number table, while the other nasolabial fold was operated on by using the same method, according to the ethical strategy of clinical trials. To minimize bias, the subjects, operators, and evaluators were blinded to the experimental side.

\section{Study device}

In this study, we used a nonabsorbable polypropylene mesh (Dmed Inc., Seoul, Korea; $12 \mathrm{~cm} \times 1.4 \mathrm{~cm}$ ) with polypropylene thread passing through it to pull the mesh for lifting (Fig. 1).

\section{Study design}

Once enrolled in the current study, all patients were evaluated by the investigators using the Wrinkle Severity Rating Scale (WSRS) and by using a self-administered visual analog scale (VAS) to determine the severity of wrinkles prior to the insertion of the study device. All subjects underwent the nonabsorbable polypropylene mesh treatment on the bilateral nasolabial folds. At each study center, the subjects were treated and evaluated by the same investigators. Thus, attempts were made to minimize bias due to differences in the treatment modality and to consistently assess efficacy throughout the study period. The study details were disclosed to the investigators who treated the patients by using a randomization chart, but they were blinded to those who assessed them. Thus, attempts were made to maintain the consistency and objectivity of assessment. At 3 weeks after the polypropylene mesh insertion, mesh lifting was performed. Patients visited the study center at 4 and 7 weeks postoperatively to evaluate the occurrence of adverse events (AEs). At 7 weeks, they also underwent clinical photography and provided a VAS score for satisfaction.

\section{Operative procedure}

This technique is divided into two independent operations. In

\section{Fig. 1. Polypropylene mesh with polypropylene thread}

A nonabsorbable polypropylene mesh (D-med Inc., Korea, $12 \mathrm{~cm} \times$ $1.4 \mathrm{~cm}$ ) with polypropylene thread passing through it to pull the mesh for lifting.

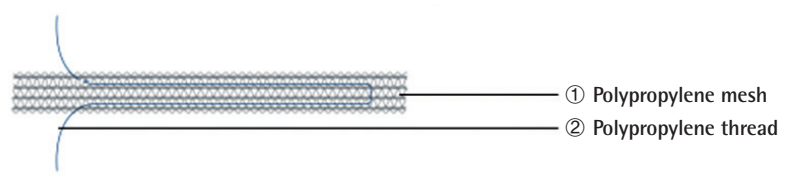

the first operation, the design was created with the subject in a seated position, and dotted marking was made with a surgical marker on the skin where the mesh would be inserted in order to determine the appropriate dissection range. Then, the patient was laid supine on the operating table with skin preparation and draping and was sedated under monitored anesthesia care. After infiltrating a local anesthetic solution (1:100,000 epinephrine mixed with $2 \%$ lidocaine), a 0.5 -cm-long incision line was made in the bilateral temporal areas, $1 \mathrm{~cm}$ inferior to the superior temporal crest and $2 \mathrm{~cm}$ behind the hairline. After the temporal incision was made, dissection was performed through the subcutaneous tissue and the superficial temporal fascia, down to the deep temporal fascia. Dissection was performed between the superficial and deep temporal fascia up to the hairline level in order to prevent alopecia of the temple and was continued to the posterior portion of the zygomatic arch (Fig. 2). Lower to the zygomatic arch, the dissection plane was located more superficially between the superficial muscular aponeurotic system and the subcutaneous level. After dissecting to the level of the nasolabial fold, the surgeon inserted a mesh through the dissection plane with a pair of specially designed forceps (Fig. 3). After the insertion, the mesh was placed in position and cut to the appropriate size while avoiding mesh kinking. The distal point of the mesh beneath the nasolabial fold level did not need to be sutured, but the proximal end was sutured to the undersurface of the scalp in order to hide the mesh. Then, the incision site was repaired using 5-0 Prolene. We did not apply any compression or bulky dressing on the cheek but did apply a small amount of antibiotic ointment at the suture site.

The second operation was performed after 3 weeks; the patient was prepared as in the initial operation, and an incision was made

\section{Fig. 2. Intraoperative photograph}

Dissection was performed through the subcutaneous tissue and the superficial temporal fascia, down to the deep temporal fascia.

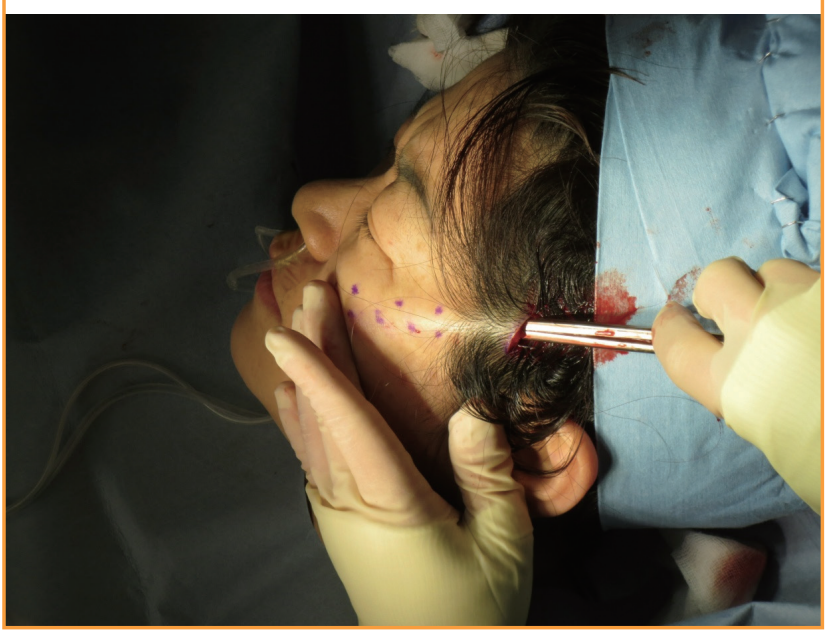


on the previous incision line. Dissection was performed through the plane previously made until the mesh attached to the soft tissue was located. Then, by using the polypropylene thread sutured to the proximal end of the mesh, the surgeon pulled the proximal end of the mesh in the cephalic direction. The direction and the traction force of the mesh were controlled to the point that the soft tissue was effectively placed for maximum tension. After ensuring the absence of a foreign body or bleeding point, the surgeon fixed the mesh to the deep temporal fascia by using nonabsorbable sutures. Finally, the scalp incision was closed in a routine manner. Ice packs were applied intermittently for a day after surgery, and the patients were given oral antibiotics for 3 days.

\section{Efficacy outcome measures}

\section{Primary outcome measure: wrinkle improvement rate}

The severity of wrinkles was evaluated using the WSRS preoperatively and at 7 weeks postoperatively by an independent evaluator (Table 2). The following formula was used: preoperative WSRS score-postoperative WSRS score.

If the result was $\geq 1$, the treatment was classified as effective (improvement of wrinkles); if the result was $<1$, the treatment was classified as ineffective (no improvement or increased wrin-

\section{Fig. 3. Photograph of polypropylene mesh and forceps}

A pair of wide forceps was specially designed for holding the mesh and inserting it through the dissection plane.

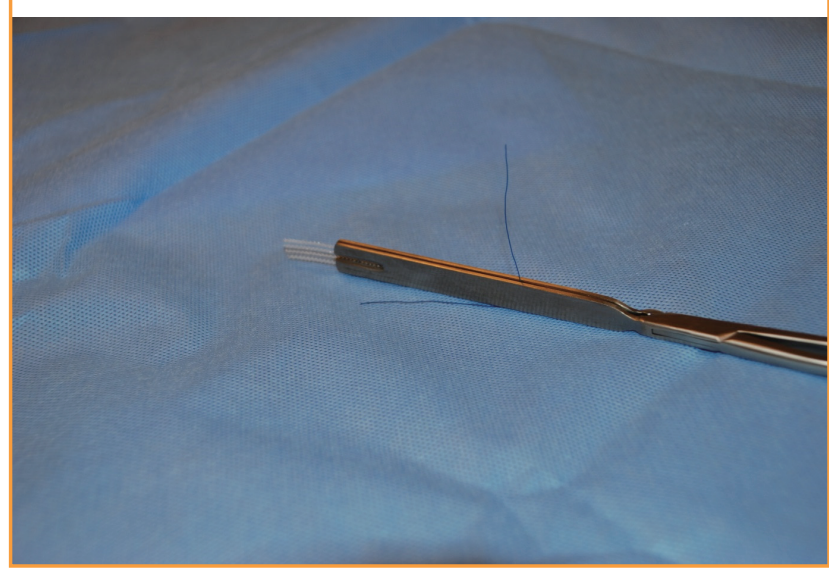

kles). Preoperative and postoperative data were analyzed statistically by using Fisher's exact test or the chi-squared test.

\section{Secondary outcome measure: patient satisfaction}

Patient satisfaction was evaluated using a self-administered VAS (0 points, no wrinkles; 100 points, severe wrinkles) preoperatively and at 7 weeks postoperatively. Preoperative and postoperative data were analyzed statistically using Wilcoxon's signedrank test.

\section{Safety analysis}

In the current study, we evaluated safety based on AEs and physical examination. AEs included tension, edema, inflammation, petechiae, erythema, tenderness, mesh rupture, inconvenience, and foreign body sensation. We evaluated all AEs that occurred in the subjects, the number of AEs, and the proportion of subjects who presented with more than one AE. We also provided the severity of each AE. In providing the frequency of AEs, we analyzed the overall frequency of AEs, adverse device events, and serious AEs.

\section{Data analysis}

Statistical analysis

In the current study, unless otherwise noted, we performed twosided tests with a statistical significance of 0.05 . In addition, we provided the number of subjects, mean, standard deviation, median, minimum, and maximum values for continuous variables, and the frequency and proportion of subjects for categorical variables. Finally, we replaced the missing values by using the worst observation carried forward method.

\section{Efficacy sets}

The data obtained were divided into full analysis (FA), per-protocol (PP), and safety sets. The main population was analyzed in the FA set, additional analysis was performed in the PP set, and safety analysis was conducted in the safety set.

Full analysis set

The FA set contained all subjects who were given a randomiza-

\section{Table 2. Wrinkle Severity Rating Scale}

\begin{tabular}{|c|c|}
\hline Score & Description \\
\hline 5 & $\begin{array}{l}\text { Extreme: extremely deep and long fold; } 2-4-\mathrm{mm} \text { visible v-shaped fold when stretched; detrimental to appearance; unlikely to have satisfactory correction with } \\
\text { injectable implant alone }\end{array}$ \\
\hline 4 & Severe: very deep and long fold; prominent facial feature; <2-mm visible fold when stretched \\
\hline 3 & Moderate: moderately deep fold; clear facial feature visible at normal appearance but not when stretched; excellent correction expected \\
\hline 2 & Mild: shallow but visible fold with slight indentation; minor facial feature \\
\hline 1 & Absent: no visible fold; continuous line \\
\hline
\end{tabular}


tion number after being enrolled in the current study. We excluded the following subjects: (1) subjects who were enrolled in the current study but did not meet the inclusion criteria at the screening visit, (2) subjects who were enrolled in the current study but did not undergo treatment, and (3) subjects who were enrolled in the current study but did not undergo the efficacy analysis.

\section{Per-protocol set}

The PP set contained all FA subjects who completed the current study without seriously violating the study protocol. We excluded the following subjects: (1) subjects who did not submit written informed consent, (2) subjects who did not undergo the efficacy analysis at 7 weeks postoperatively, (3) subjects who underwent procedures or treatments during the study period that might affect the results of the efficacy analysis (including prohibited concomitant medications), and (4) subjects who seriously violated the study protocol according to the judgment of the investigators.

\section{Safety set}

The safety set comprised all subjects who were enrolled in the current study and underwent a safety analysis after the treatment.

\section{RESULTS}

\section{Baseline characteristics}

In the current study, we recruited a total of 33 subjects, but 4 were excluded through baseline screening and 1 withdrew written informed consent. Therefore, 28 patients underwent the procedure; all these patients were female with an average age of 54 years (range, 45-65 years). One patient withdrew written in-

\section{Fig. 4. Wrinkle improvement rate}

If (preoperative WSRS score-postoperative WSRS score) $>1$, the treatment was classified as effective, and if the result was $\leq 1$, the treatment was classified as ineffective. WSRS, Wrinkle Severity Rating Scale; FA, full analysis; PP, per-protocol.

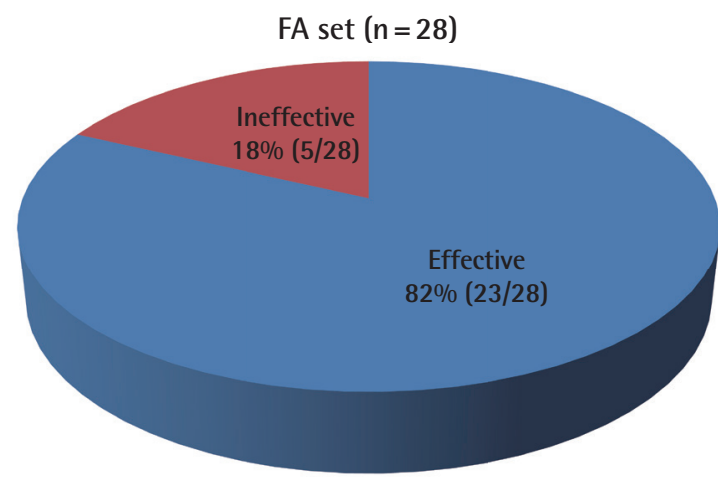

formed consent after the procedure, so we evaluated a total of 27 subjects who completed the study within the study period. The number of patients in the FA, PP, and safety sets were 28, 25 , and 27 , respectively.

\section{Efficacy outcomes}

An efficacy evaluation was conducted in the FA set $(n=28)$, while a secondary analysis was carried out in the PP set $(n=25)$. The treatment was deemed to be effective at improving wrinkles in 23 of 28 cases ( $82.14 \%$ ) and ineffective in 5 cases (17.86\%). In the PP set analysis, the treatment was deemed to be effective in 23 of 25 cases (92.0\%) and ineffective in 2 cases (8.0\%) (Fig. 4).

As a secondary outcome measure, patient satisfaction was evaluated according to the VAS score. In the FA set, the mean preoperative VAS score was $70.71 \pm 16.54$ (range, 20-90), while the mean postoperative VAS score was $46.07 \pm 18.12$ (range, $20-80)$; this difference was statistically significant $(\mathrm{P}<0.001)$. In the PP set analysis, the mean preoperative VAS score was $73.20 \pm 13.76$ (range, 30-90), while the mean postoperative VAS score was $45.60 \pm 17.58$ (range, 20-80); this difference was also statistically significant $(\mathrm{P}<0.001)$ (Fig. 5). Finally, in both the FA and the PP sets, patient satisfaction increased significantly after the operation (Figs. 6, 7).

\section{Safety outcomes}

Among the FA set, 1 patient was lost to follow-up and 27 patients were examined for abnormal response at 4 and 7 weeks after surgery. According to the system organ class, there were seven cases of skin or subcutaneous tissue complications at 7 weeks postoperatively; they included edema $(22.22 \%, 6 / 27)$ and ery-

\section{Fig. 5. Patient satisfaction}

Patient satisfaction was evaluated according to the difference between the preoperative and the postoperative visual analog scale (VAS) scores. FA, full analysis; PP, per-protocol.

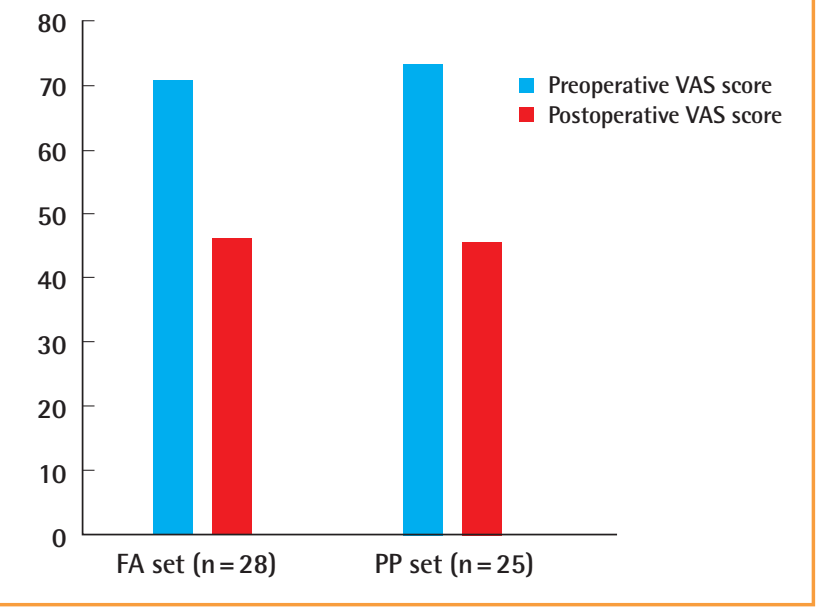


Fig. 6. A case of a 53-year-old female
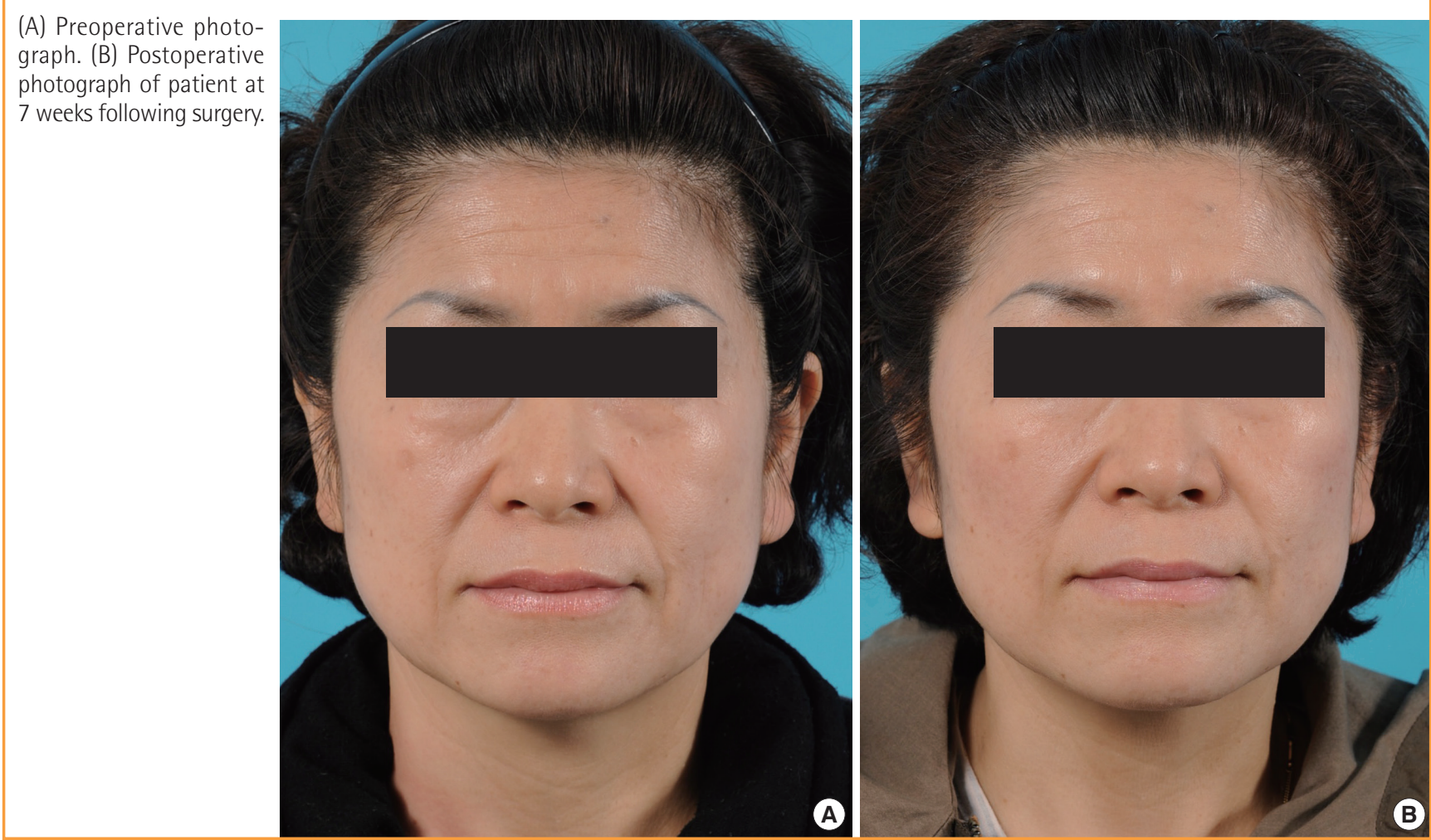

Fig. 7. A case of a 51-year-old female

(A) Preoperative photograph. (B) Postoperative photograph of patient at

7 weeks following surgery.
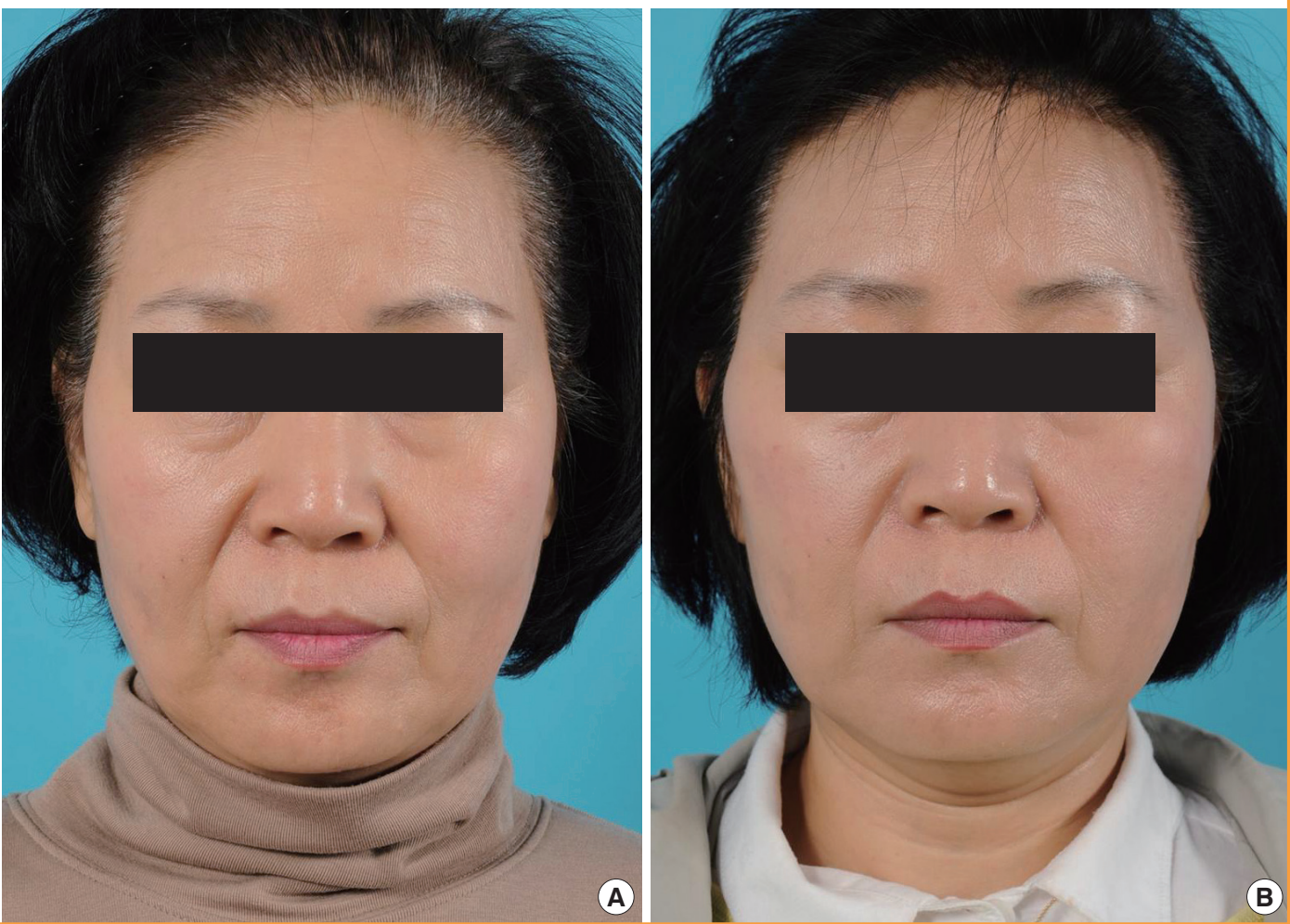
thema $(3.70 \%, 1 / 27)$. During the study period, there were no suspected serious AEs.

\section{DISCUSSION}

Face lifting alternatives can be categorized into two types based on the technique used-square-shaped template insertion or thread insertion-even though they have the same purpose of insertion and lifting. Square-shaped template insertion can also be further divided into two types based on the material used: absorbable (Vicryl mesh) or nonabsorbable (Prolene mesh or Gore-tex mesh). In 2000, Stuzin et al. [8] reported the elevation of the superficial muscular aponeurotic system flap in conventional face lifting surgery by the fixation and suspension of the flap by using a Vicryl mesh. This fixation improved the contour of the midface and safely repositioned the facial fat. Gore-tex insertion, as a nonabsorbable square-shaped template, was used in face lifting procedures by Kim et al. [9], who reported the rejuvenation of the neck and lower face following two different staged operations: insertion of the Gore-tex (first) and lifting procedure (second). These two-stage operations are the same as those in the case of a polypropylene mesh. The first operation consists of the insertion of the template, followed by several weeks of waiting for template adhesion with the adjacent tissue. In the second surgery, the template had sufficient adhesive force to be lifted and pulled in the desired direction. However, the thickness of the Gore-tex implant was $0.64 \mathrm{~mm}$, while that of the polypropylene mesh is $0.1 \mathrm{~mm}$. The thickness of the lower chin skin in Korean patients is $0.857 \pm 0.247 \mathrm{~mm}$ [10]; thus, the insertion of the Gore-tex template is palpable.

Polypropylene meshes are used in many fields. For example, Sampaio reported the use of a mesh supporting system in a double-skin technique for mammoplasty, and in the closure of the abdominal wall in numerous other operations [11]. In addition, Kwon et al. [12] proved the mechanical strength and stretching force of a polypropylene mesh to be sufficient for use in brow suspension. Mutaf [7] also reported the use of a polypropylene mesh in brow lifting without any foreign body reaction or inflammation during a long-term clinical observation.

As a face lifting alternative, thread lifting has also become popular in Asia. Silhouette lifting, composed of 3-0 Prolene thread and an absorbable cone, has many advantages, including short operating time and the use of simple local anesthesia [13]. However, its drawbacks are its longevity and revision procedure after relapse. These minimally invasive surgical procedures have some shortcomings, such as limited durability and relapse, but they are popular because of their simplicity and rapid recovery time. The mean operating time for such procedures is $30-60$ minutes, and surgery can be performed at minimal anesthetic depth; thus, patients can return to their social life quickly, with minimal swelling and tenderness.

Despite these advantages, there are some possible AEs following polypropylene mesh lifting surgery. Unlike the thread lifting method, mesh lifting requires a skin incision and the dissection of a wide area for skin contact. Further, after 3 weeks, a lifting procedure including the dissection of the skin-mesh adhesion is required. All these procedures can increase the rate of tenderness, inflammation, swelling, and foreign body sensation compared with the use of the thread lifting method. Furthermore, foreign body-related complications, foreign body granuloma, inflammatory response, and exposure of the mesh can occur. Kim et al. reported wound infection in 12 cases (2.7\%) after the insertion of a Gore-tex template during the follow-up period. We did not encounter any inflammatory response during the two-month follow-up period.

For mesh lifting, appropriate patient selection is important. Mesh lifting is helpful in mild-to-moderate cases. It is difficult to apply the mesh in patients with severe skin laxity and wrinkles, since massive skin redundancy cannot be corrected. This procedure should also be avoided in patients with very thin skin, since the implant can be palpable even if it is very thin (e.g., $0.1 \mathrm{~mm}$ ). Patients with a puffy face or thick, sebaceous skin should avoid the procedure because lifting is very difficult after the insertion of the mesh. The requisite two-stage operation is the definite shortcoming of mesh lifting. Patients can be burdened by the two operations, even though each procedure is very simple and takes a short amount of time. In all cases, sufficient preoperative counseling is important. As in conventional face lifting surgery, facial asymmetry can occur after mesh lifting, which can be corrected by additional lifting procedures. In this study, there were 7 cases of skin or subcutaneous tissue AEs at 4 weeks after surgery; they included edema $(22.22 \% ; 6 / 27)$ and erythema (3.70\%; $1 / 27)$. These effects disappeared at the end of surgery, and polypropylene mesh lifting was proven to be safe during the study and the follow-up periods.

In this clinical trial, we created a pair of special forceps for the application of the mesh (Fig. 3). After dissection of the flap, these forceps were used to insert the mesh safely without any kinking or insufficient placement. After insertion, a needle was applied through the skin to fix the mesh, and the forceps were removed. Folding of the mesh during the insertion procedure may allow it to be palpable and cause an insufficient adhesive contact surface with the surrounding tissue. After fixation of the mesh with 3-0 Prolene, the knot of the stitches is concealed into the deep temporal fascia to prevent any foreign body granuloma. During the dissection of the flap, the surgeon should try to avoid cutting the 
tissue so as not to damage the nerve branches. Even if nerve damage occurs, recovery is still certain. The mesh should be placed near the nasolabial fold to achieve sufficient lifting; inappropriate placement can result in an insufficient lifting effect or an unnatural look of the face. Polypropylene mesh lifting is useful for patients who do not want to undergo conventional invasive face lifting surgery. Based on these results, we infer that minimal bruising and swelling, and quick return to normal social life are the major advantages of polypropylene mesh lifting.

However, this clinical trial has several limitations, including the small number of subjects and the relatively short follow-up period, but this is the first clinical trial to evaluate the efficacy and safety of the use of a polypropylene mesh in midface lifting surgery. Despite these limitations, we demonstrated that the use of a polypropylene mesh is safe and effective for midface lifting as a successful alternative to conventional face lifting surgery.

\section{REFERENCES}

1. Abraham RF, DeFatta RJ, Williams EF 3rd. Thread-lift for facial rejuvenation: assessment of long-term results. Arch Facial Plast Surg 2009; 11:178-83.

2. Savoia A, Accardo C, Vannini F, et al. Outcomes in thread lift for facial rejuvenation: a study performed with happy lift revitalizing. Dermatol Ther (Heidelb) 2014;4:103-14.

3. Shin KC, Bae TH, Kim WS, et al. Usefulness of gold thread implantation for crow's feet. Arch Plast Surg 2012;39:42-5.

4. Citarella ER, Sterodimas A, Green AC, et al. Use of tripleconvergence polypropylene thread for the aesthetic correction of partial facial paralysis. Aesthetic Plast Surg 2008;32:
688-91.

5. Park TH, Seo SW, Whang KW. Facial rejuvenation with finebarbed threads: the simple Miz lift. Aesthetic Plast Surg 2014; 38:69-74.

6. de Benito J, Pizzamiglio R, Theodorou D, et al. Facial rejuvenation and improvement of malar projection using sutures with absorbable cones: surgical technique and case series. Aesthetic Plast Surg 2011;35:248-53.

7. Mutaf M. Mesh lift: a new procedure for long-lasting results in brow lift surgery. Plast Reconstr Surg 2005; 116:1490-9.

8. Stuzin JM, Baker TJ, Baker TM. Refinements in face lifting: enhanced facial contour using vicryl mesh incorporated into SMAS fixation. Plast Reconstr Surg 2000;105:290-301.

9. Kim JM, Jang YJ, Kwon BS, et al. Lower face-lift with expanded polytetrafluoroethylene superficial musculoaponeurotic system extension graft (bandlift implant). J Craniofac Surg 2010;21:1928-31.

10. Lee Y, Hwang K. Skin thickness of Korean adults. Surg Radiol Anat 2002;24:183-9.

11. Moscona RA, Ramon Y, Toledano $\mathrm{H}$, et al. Use of synthetic mesh for the entire abdominal wall after TRAM flap transfer. Plast Reconstr Surg 1998;101:706-10.

12. Kwon KA, Shipley RJ, Edirisinghe M, et al. Microstructure and mechanical properties of synthetic brow-suspension materials. Mater Sci Eng C Mater Biol Appl 2014;35:22030.

13. Isse N. Silhouette sutures for treatment of facial aging: facial rejuvenation, remodeling, and facial tissue support. Clin Plast Surg 2008;35:481-6. 